\title{
Nocturnal saturation and glucose tolerance in children with cystic fibrosis
}

\author{
Dharmeshkumar Suratwala, ${ }^{1}$ June S H Chan, ${ }^{2}$ Andrea Kelly, ${ }^{3}$ Lisa J Meltzer, ${ }^{1}$ \\ Paul R Gallagher, ${ }^{4}$ Joel Traylor, ${ }^{1}$ Ronald C Rubenstein, ${ }^{1}$ Carole L Marcus ${ }^{1,4}$
}

- Additional materials are published online only. To view these files please visit the journal online (http://thorax.bmj. com).

${ }^{1}$ Division of Pulmonary

Medicine, Cystic Fibrosis and Sleep Center, Children's

Hospital of Philadelphia, University of Pennsylvania School of Medicine, Philadelphia, Pennsylvania, USA ${ }^{2}$ Department of Paediatrics and Adolescent Medicine, Pamela Youde Nethersole Eastern Hospital, Hong Kong ${ }^{3}$ Division of Endocrinology, Children's Hospital of Philadelphia, University of Pennsylvania School of Medicine, Philadelphia, Pennsylvania, USA

${ }^{4}$ Clinical and Translational Research Center, Children's Hospital of Philadelphia University of Pennsylvania School of Medicine, Philadelphia, Pennsylvania, USA

\section{Correspondence to}

Carole L Marcus, Children's Hospital of Philadelphia, Pulmonary Division, 9NW50, 34th Street \& Civic Center Boulevard, Philadelphia, PA 19104, USA; marcus@email. chop.edu

DS and JSHC contributed equally to this work.

Received 6 May 2010 Accepted 16 December 2010 Published Online First 27 January 2011

\section{ABSTRACT}

Background Glucose intolerance is common in cystic fibrosis (CF), and is associated with worsening pulmonary function and nutritional status, and increased mortality. As sleep-disordered breathing is associated with disorders of glucose metabolism, it was hypothesised that recurrent episodes of hypoxaemia during sleep, and sleep disruption, would be associated with inflammation and glucose intolerance in CF. Methods 25 children (aged $14 \pm 4$ (mean \pm SD) years) with CF underwent polysomnography, actigraphy, measurement of serum inflammatory markers and oral glucose tolerance testing. Blood glucose area under the curve (AUC), as a cumulative measure of glucose response, was determined. Polysomnography data were compared with retrospective data from 25 healthy controls

Results Forced expiratory volume in $1 \mathrm{~s}$ was $92 \pm 14 \%$ predicted. 24 subjects underwent glucose tolerance testing, of whom $29 \%$ had impaired glucose tolerance and $4 \%$ had diabetes. The mean nocturnal oxygen saturation correlated negatively with glucose AUC at $120 \min (r=-0.49, p=0.015)$. Partial correlations and regression models including age, body mass index, nocturnal saturation and pulmonary function indicated that nocturnal saturation accounted for the majority of the predictive power for glucose $A \cup C\left(R^{2}=0.24\right.$, $p=0.001)$. There were no meaningful relationships between sleep quality, inflammation and glucose tolerance.

Conclusions Lower oxyhaemoglobin saturation is associated with worse glucose regulation in children with CF. Further studies are needed to determine whether lower saturation negatively impacts glucose regulation or, alternatively, whether abnormalities in glucose metabolism are an early sign of pulmonary dysfunction.

\section{INTRODUCTION}

Cystic fibrosis (CF) is a common genetic disease with significant morbidity and mortality. With improved survival as well as improved screening practices, cystic fibrosis-related diabetes mellitus (CFRD) has emerged as a common complication of CF. ${ }^{1}$ Insulin deficiency is the primary defect in CFRD, although a variable degree of insulin resistance does occur. ${ }^{2}$ CFRD is associated with a more rapid decline in pulmonary status, worse nutritional status and increased mortality. ${ }^{4}$ However, the underlying pathophysiology linking CFRD and pulmonary function has not been fully elucidated.

Sleep-disordered breathing and subclinical inflammation have recently been shown to play an important role in the development of insulin resistance and glucose intolerance in non-CF populations. ${ }^{5}$ Sleep-disordered breathing may affect glucose metabolism by various mechanisms including sympathetic hyperactivity secondary to sleep fragmentation, sleep loss and hypoxaemia ${ }^{6}$; altered regulation of the hypothalamic-pituitaryadrenal axis ${ }^{7}$; and/or direct effects of hypoxaemia on glucose homeostasis and insulin secretion. ${ }^{8}$ Patients with CF are prone to impaired sleep quality and sleep-related hypoxaemia due to nocturnal cough, nasal obstruction due to chronic infection or nasal polyps, and hypoxaemia and increased work of breathing related to lower airway obstruction. $^{9} 10$

Sleep-disordered breathing is also associated with inflammation. Patients with sleep-disordered breathing have been reported to have elevated C-reactive protein $(\mathrm{CRP})^{11}$ and activation of nuclear factor- $\kappa \mathrm{B}(\mathrm{NF}-\kappa \mathrm{B}){ }^{12}$ resulting in increased circulating levels of inflammatory cytokines including interleukin 6 (IL-6), ${ }^{13}$ IL-8, tumour necrosis factor $\alpha$ (TNF $\alpha)$, soluble E-selectin (sE-selectin), soluble vascular cell adhesion molecule-1 (sVCAM-1) ${ }^{14}$ and intracellular adhesion molecule-1 (ICAM-1). ${ }^{15}$ Sleep deprivation also results in elevation of CRP, ${ }^{16}$ TNF $\alpha$ and IL- $6 .{ }^{17}$ Inflammatory markers, including CRP, IL-6 and sVCAM-1, likewise are increased with glucose intolerance. ${ }^{18}$ Patients with CF have ongoing infection and neutrophilic inflammation in their airways. They may also have elevated circulating proinflammatory cytokines at baseline, even during exacerbation-free periods, ${ }^{19}$ although other inflammatory markers such as CRP are more variable. Patients with CF with impaired glucose tolerance and CFRD have significantly higher levels of TNF $\alpha$ compared with patients with CF with normal glucose tolerance, ${ }^{3}$ as well as a more rapid rate of pulmonary function (forced expiratory volume in $\left.1 \mathrm{~s}\left(\mathrm{FEV}_{1}\right)\right)$ decline. ${ }^{20}$ which may reflect the negative impact of hyperglycaemia on neutrophil function. In addition, patients with CF may be more prone to sleep-disordered breathing due to chronic sinus disease, nasal polyposis and/or increased cough frequency. Thus, it is possible that there is an association, and possibly even a causal relationship, between sleep-disordered breathing and these inflammatory biomarkers, and hence impaired glucose tolerance, in patients with CF

We hypothesised that recurrent episodes of hypoxaemia during sleep and sleep disruption (poor sleep efficiency and quality) would be associated with worse glucose tolerance in patients with CF, as well as increased biomarkers of systemic inflammation. We therefore evaluated sleep characteristics 
(using both polysomnography and actigraphy), glucose tolerance and inflammatory biomarkers in patients with CF. Polysomnographic parameters were compared with retrospective data from healthy controls.

\section{METHODS}

Additional details of the methods for making these measurements is provided in an online data supplement.

Stable subjects with CF, aged 8-21 years of age, underwent pulmonary function testing, overnight polysomnography, the Epworth Sleepiness Scale (modified for children), ${ }^{21}$ glucose tolerance testing and measurement of serum inflammatory markers (CRP, TNF, IL-1 $\beta$, IL-6, IL-8, IL-10, sE-selectin, sVCAM and sICAM) the morning following polysomnography, and 14 days of home actigraphy. Control subjects were obtained retrospectively from a normal control database of healthy individuals recruited from the community for polysomnographic studies.

The Institutional Review Board of the Children's Hospital of Philadelphia approved the study. Written informed consent was obtained from subjects 18 years of age or older, and from the parent or legal guardian of younger subjects. Assent was obtained from subjects under 18 years of age.

\section{Study group}

Subjects with CF $\geq 8$ years of age were recruited from the Cystic Fibrosis Center of the Children's Hospital of Philadelphia. Subjects were excluded if they had a known history of diabetes mellitus, were pregnant, had acute illness or had used medications (including systemic steroids) known to affect insulin sensitivity within the past 6 weeks, had a history of organ transplantation, a body mass index (BMI) >85th percentile, history of severe liver disease or chronic use of supplemental home oxygen or non-invasive positive pressure ventilation. For polysomnographic data, each subject was gender and agematched to a normal control subject from our database. To determine whether the study group was representative of the CF Center population at our institution, comparisons were also made between study subjects and all patients in that age range (8-21 years) in our CF Center database.

\section{Polysomnography}

All subjects underwent standard paediatric polysomnography. ${ }^{22}$ Arterial oxygen saturation $\left(\mathrm{SpO}_{2}\right)$ was measured by pulse oximetry (Nonin Medical, Plymouth, Minnesota, USA), and the oximeter pulse waveform was recorded.

\section{Oral glucose tolerance test (OGTT)}

A glucose tolerance test was performed after overnight fasting. Impaired glucose tolerance was defined as a $2 \mathrm{~h}$ plasma glucose of 140-199 mg/dl; CFRD without fasting hyperglycaemia as a $2 \mathrm{~h}$ plasma glucose $\geq 200 \mathrm{mg} / \mathrm{dl}$, and indeterminate glucose tolerance as a normal $2 \mathrm{~h}$ plasma glucose but a 60 min glucose $>200 \mathrm{mg} / \mathrm{dl}^{23}$ The inter-relationships between demographic, polysomnographic and actigraphic parameters, biomarkers and blood glucose parameters (primarily blood glucose area under the curve (AUC)) were evaluated. Glucose AUC was chosen to characterise glucose status further because the insulin secretory defects which typify CF lead to increases in glucose excursion following a glucose load. ${ }^{24}$ The normal glucose tolerance, impaired glucose tolerance and CFRD categories are based only on the $2 \mathrm{~h}$ blood glucose, and do not adequately capture the breadth of these glucose abnormalities for study purposes. Like continuous glucose monitoring, OGTT glucose AUC and OGTT blood glucose levels obtained earlier than 120 min frequently identify glucose excursions in the setting of CF that are not captured by the $2 \mathrm{~h}$ OGTT blood glucose. ${ }^{25}$ Total glucose AUC for plasma glucose from 0 to $120 \mathrm{~min}$ was therefore calculated. As glucose intolerance worsens with increasing age and is associated with lower BMI in patients with CF, the relationship between blood glucose AUC and mean nocturnal $\mathrm{SpO}_{2}$, controlling for age and BMI z-score, was explored using partial correlation coefficients and linear regression.

\section{Actigraphy}

Each subject wore an actigraph (Actiwatch-64, Mini-Mitter, Respironics, Bend, Oregon, USA) on the non-dominant wrist for 14 days following the polysomnogram and glucose tolerance test. Subjects or their caregiver completed a simultaneous sleep diary.

\section{Data analysis}

Details of the data analytic methods are provided in the online supplement. Histograms and Kolmogorov-Smirnov tests were used to examine the distributions of continuous outcome variables. Demographic variables, pulmonary function parameters and polysomnographic parameters were compared between subjects with CF and controls using appropriate statistical tests: independent sample $t$ tests were used to compare parametric variables, and Mann-Whitney tests were used to compare nonparametric variables. The Fisher exact test was used to compare the groups on gender. To control for multiple comparisons, a $p$ value $<0.017$ was considered significant. Within the group with CF, bivariate correlations, partial correlations and linear regression models were used to assess the relationships between demographic, polysomnographic and actigraphic parameters, biomarkers and blood glucose AUC.

\section{RESULTS}

Twenty-five subjects with CF were studied (table 1). The study population was comparable with the overall CF Center population aged 8-21 years, which had an $\mathrm{FEV}_{1}$ of $97 \pm 19 \%$ predicted $(p=0.10$ compared with study subjects), a mean BMI percentile of $60.5 \pm 23.4$ (compared with $55.4 \pm 26.7$ for study subjects, $\mathrm{p}=0.40$ ) and a rate of impaired glucose tolerance of $17 \%$ $(p=0.37)$ and CFRD of $2 \%(p=0.55)$ as observed during routine OGTT in otherwise well patients in the outpatient setting.

Spirometry in the study group was consistent with normal lung function or mild obstructive lung disease, and the mean Epworth Sleepiness Scale was normal (table 1). ${ }^{21}$

\section{Polysomnography results}

Polysomnography results are shown in table 1 . Subjects with CF had a higher arousal index than controls, but sleep architecture was otherwise similar. The mean $\mathrm{SpO}_{2}$ was significantly lower in subjects with CF compared with controls, although values were still in the clinically normal range.

\section{Actigraphy results}

Actigraphic data were obtained for 21 subjects as four subjects failed to return the device. A total of $12 \pm 4$ nights/subject were fit for analysis. The sleep period was $440 \pm 54 \mathrm{~min}$, sleep efficiency $80 \pm 7 \%$ and waking after sleep onset $83 \pm 35 \mathrm{~min}$.

\section{Glucose tolerance}

Twenty-four subjects with CF underwent OGTT; one subject refused phlebotomy. Mean fasting plasma glucose was $96 \pm 10 \mathrm{mg} / \mathrm{dl}$ and mean $2 \mathrm{~h}$ glucose was $129 \pm 34 \mathrm{mg} / \mathrm{dl}$. One (4\%) subject was classified as having CFRD without fasting hyperglycaemia, 7 (29\%) subjects with impaired glucose 
Table 1 Demographic and polysomnographic data from subjects with cystic fibrosis and controls

\begin{tabular}{|c|c|c|c|}
\hline & Cystic fibrosis & Controls & p Value \\
\hline Subjects (n) & 25 & 25 & - \\
\hline Age, years (range) & $14 \pm 4(8-20)$ & $14 \pm 4(7-20)$ & 0.77 \\
\hline Male (n) & 15 & 15 & 1.00 \\
\hline BMI z-score & $0.1 \pm 0.9$ & $0.4 \pm 0.8$ & 0.32 \\
\hline Epworth Sleepiness Scale & $5 \pm 4$ & NA & - \\
\hline \multicolumn{4}{|l|}{ Pulmonary function parameters } \\
\hline $\begin{array}{l}\text { Forced vital capacity (FVC), } \\
\% \text { predicted }\end{array}$ & $99 \pm 12$ & NA & - \\
\hline $\begin{array}{l}\text { Forced expiratory volume in } \\
1 \mathrm{~s}\left(\mathrm{FEV}_{1}\right), \% \text { predicted }\end{array}$ & $92 \pm 14$ & NA & - \\
\hline $\mathrm{FEV}_{1} / \mathrm{FVC}(\%)$ & $81 \pm 9$ & NA & - \\
\hline \multicolumn{4}{|l|}{ Polysomnographic parameters } \\
\hline Total recording time, $\min$ & $522 \pm 75$ & $510 \pm 30$ & 0.74 \\
\hline Sleep efficiency, \% TST & $78 \pm 13$ & $83 \pm 13$ & 0.13 \\
\hline Total sleep time, min & $383 \pm 62$ & $412 \pm 69$ & 0.13 \\
\hline Wake after sleep onset, min & $83 \pm 68$ & $55 \pm 51$ & 0.11 \\
\hline Sleep latency, min & $31 \pm 32$ & $27 \pm 18$ & 0.57 \\
\hline Latency to REM, min & $128 \pm 71$ & $132 \pm 74$ & 0.82 \\
\hline Stage N1, \% TST & $9 \pm 4$ & $7 \pm 4$ & 0.10 \\
\hline Stage N2, \% TST & $50 \pm 8$ & $53 \pm 7$ & 0.20 \\
\hline Stage N3, \% TST & $22 \pm 10$ & $22 \pm 8$ & 0.85 \\
\hline REM, \% TST & $19 \pm 5$ & $18 \pm 5$ & 0.73 \\
\hline Arousal index, $\mathrm{N} / \mathrm{h}$ & $16 \pm 5$ & $11 \pm 4$ & $<0.001$ \\
\hline Obstructive apnea index, N/h & $0.0(0.0,0.6)$ & $0.0(0.0,0.2)$ & 0.13 \\
\hline Hypopnoea index, N/h & $0.4(0.0,6.0)$ & $0.0(0.0,0.9)$ & 0.066 \\
\hline $\mathrm{SpO}_{2}$ mean, $\%$ & $96.6 \pm 1.0$ & $97.5 \pm 0.7$ & $<0.001$ \\
\hline $\mathrm{SpO}_{2}$ nadir, $\%$ & $92.5 \pm 2.2$ & $93.8 \pm 1.9$ & 0.031 \\
\hline Time with $\mathrm{SpO}_{2}<92 \%$, \% TST & $0.0(0.0,6.7)$ & $0.0(0.0,0.2)$ & 0.11 \\
\hline $\mathrm{TcCO}_{2}$ mean, $\mathrm{mm} \mathrm{Hg}$ & $47.8 \pm 6.8$ & NA & - \\
\hline Periodic limb movement index, $\mathrm{N} / \mathrm{h}$ & $0.0(0.0,22.8)$ & $0.0(0.0,8.7)$ & 0.32 \\
\hline
\end{tabular}

Data are presented as mean \pm SD for normally distributed variables and median (range) for skewed variables.

BMI, body mass index; NA, not available; REM, rapid eye movement sleep; $\mathrm{TcCO}_{2}$, transcutaneous $\mathrm{CO}_{2}$; TST, total sleep time.

tolerance and 16 (67\%) with normal glucose tolerance. Five of the subjects with 'normal glucose tolerance' as defined by the $2 \mathrm{~h}$ glucose level had plasma glucose $\geq 200 \mathrm{mg} / \mathrm{dl}$ at $60 \mathrm{~min}$ and were labelled at indeterminate. Mean glucose AUC from 0 to $120 \mathrm{~min}$ of the OGTT was $17650 \pm 3020 \mathrm{mg} / \mathrm{dl} / 120 \mathrm{~min}$.

\section{Biomarkers}

The values of the circulating inflammatory biomarkers from the 24 subjects, and their comparison with normative data, are presented in table 2. Although a large degree of variability was present, in general the biomarker levels were increased or at the upper level of normal compared with normative data.

\section{Factors predicting glucose intolerance}

The inter-relationships between demographic, polysomnographic and actigraphic parameters, biomarkers and blood glucose parameters (primarily blood glucose AUC) were evaluated. There was a significant negative correlation between nocturnal mean $\mathrm{SpO}_{2}$ and glucose AUC $(\mathrm{r}=-0.49, \mathrm{p}=0.015)$ (figure 1). There were two outliers that were $>2 \mathrm{SDs}$ from the mean: one subject with the lowest $\mathrm{SpO}_{2}$ and highest glucose AUC; and another with the lowest glucose AUC. Even after exclusion of these outliers, the correlation remained significant $(p=0.011)$. No significant associations were found between other polysomnographic variables, actigraphic sleep variables or biomarkers and glucose tolerance.

The relationship between blood glucose AUC and mean nocturnal $\mathrm{SpO}_{2}$, controlling for age and BMI z-score, was
Table 2 Values of inflammatory biomarkers in 24 subjects with cystic fibrosis compared with normative data

\begin{tabular}{lcl}
\hline & $\begin{array}{l}\text { Current study } \\
\text { median (range) }\end{array}$ & $\begin{array}{l}\text { Normative } \\
\text { data* }^{*} \text { (range) }\end{array}$ \\
\hline $\mathrm{CRP}, \mathrm{ng} / \mathrm{ml}$ & $1554(45-27172)$ & $120-4492$ \\
$\mathrm{TNF} \alpha, \mathrm{pg} / \mathrm{ml}$ & $2.1(1.3-25.6)$ & $0.57-1.41$ \\
$\mathrm{IL}-1 \beta, \mathrm{pg} / \mathrm{ml}$ & $0.2(0.1-0.9)$ & $\mathrm{ND}-0.22$ \\
$\mathrm{IL}-6, \mathrm{pg} / \mathrm{mL}$ & $3.6(0.3-16.1)$ & $0.43-8.87$ \\
$\mathrm{IL}-8, \mathrm{pg} / \mathrm{ml}$ & $2.9(1.1-96.5)$ & $2.5-7.2$ \\
$\mathrm{IL}-10, \mathrm{pg} / \mathrm{ml}$ & $0.7(0.0-16.2)$ & $\mathrm{ND}-35$ \\
$\mathrm{sE}-\mathrm{Selectin}, \mathrm{ng} / \mathrm{ml}$ & $54.5(17.7-105.3)$ & $15.6-66.4$ \\
$\mathrm{sVCAM}, \mathrm{ng} / \mathrm{ml}$ & $799(482-1859)$ & $301-875$ \\
sICAM, ng/ml & $310(122-435)$ & $106-337$ \\
\hline
\end{tabular}

*Normative ranges provided by the laboratory kit manufacturers.

CRP, C-reactive protein; IL, interleukin; ND, not detectable; sE-selectin, soluble E-selectin; sICAM, soluble intracellular adhesion molecule; sVCAM, soluble vascular cell adhesion molecule; TNF $\alpha$, tumour necrosis factor $\alpha$.

explored. $\mathrm{SpO}_{2}$ was negatively correlated with glucose AUC after accounting for age and BMI $z$-score $(r=-0.45, p=0.034)$. Although age was not significantly correlated with blood glucose AUC $(r=0.28, p=0.18)$, this mild correlation with age was drastically reduced and approached zero after controlling for $\mathrm{SpO}_{2}$. Conversely, controlling for $\mathrm{SpO}_{2}$ increased the mild correlation between BMI z-score and blood glucose AUC $(r=-0.36, p=0.092)$. These findings were supported by a linear regression model of blood glucose AUC in which all three predictors were introduced into the model (table 3).

Multiple linear regressions were further used to find the linear models that best fit the glucose tolerance outcome data. Best subsets regression was used to help determine which predictor variables should be included in a multiple regression model, using adjusted $\mathrm{R}^{2}$ to check for the best model. Consistently appearing among the best models for glucose tolerance outcomes were mean $\mathrm{SpO}_{2}$ and $\mathrm{BMI}$ z-score. $\mathrm{FEV}_{1} \%$ predicted was found to act as a suppressor covariate (ie, a covariate that was unrelated to the outcome variable but increased the overall model fit within regression when added to the model). In this case, $\mathrm{FEV}_{1}$ $\%$ predicted was not significantly correlated with blood glucose AUC ( $r=0.14, p=0.51)$, but was positively correlated with $\mathrm{SpO}_{2}$ $(r=0.44, p=0.028)$, which in turn was negatively correlated with blood glucose AUC ( $r=-0.49, p=0.015)$.

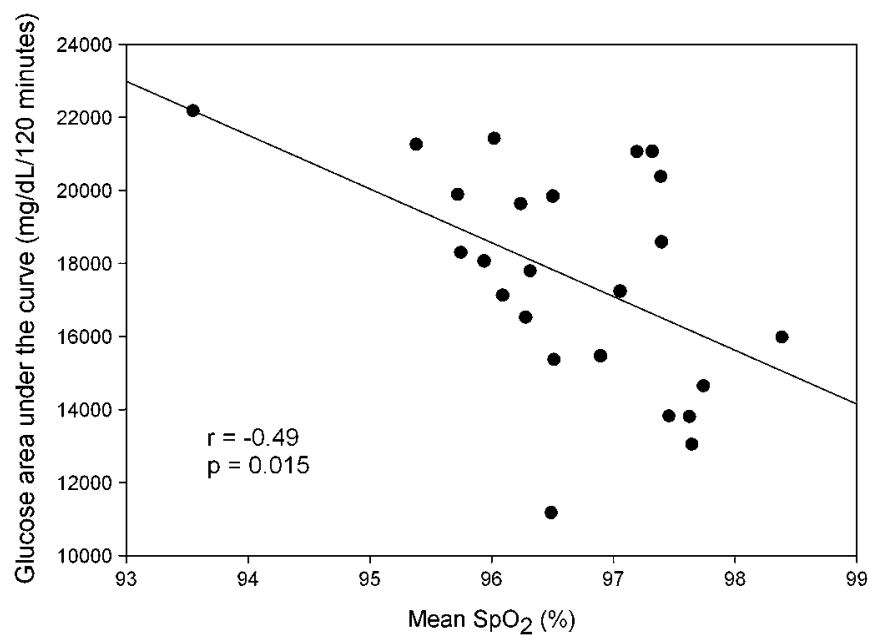

Figure 1 The correlation between nocturnal mean arterial oxygen saturation and glucose area under the curve at $120 \mathrm{~min}$ in 24 subjects with cystic fibrosis. 
Table 3 Linear regression examining the effects of age, body mass index (BMI) Z-score and $\mathrm{SpO}_{2}$ on blood glucose area under the curve at 120 min $\left(R^{2}=0.34\right)$ in 24 subjects with cystic fibrosis

\begin{tabular}{lcrllll}
\hline Predictor & $\begin{array}{l}\text { Unstandardised } \\
\boldsymbol{\beta} \text { coefficient }\end{array}$ & SE & $\begin{array}{l}\mathbf{p} \\
\text { Value }\end{array}$ & $\begin{array}{l}\text { Zero-order } \\
\text { correlation }\end{array}$ & $\begin{array}{l}\text { Partial } \\
\text { correlation }\end{array}$ & Tolerance \\
\hline Constant & 171162.9 & 63729.9 & 0.014 & & & \\
Age & -7.2 & 145.2 & 0.961 & 0.285 & -0.011 & 0.709 \\
BMI z-score & -1143.7 & 666.5 & 0.102 & -0.261 & -0.358 & 0.975 \\
Mean SpO $_{2}$ & -1585.1 & 647.8 & 0.024 & -0.492 & -0.480 & 0.704
\end{tabular}

Zero-order correlation is a measure of linear association between two variables.

Partial correlation is the correlation that remains between two variables after removing the correlation that is due to their mutual association with other variables.

Tolerance indicates the percentage variance in a predictor that could not be accounted for by the other predictors.

Using a potentially more clinically relevant approach, we sought a mean nocturnal $\mathrm{SpO}_{2}$ threshold at which traditionally defined glucose abnormalities ( $2 \mathrm{~h}$ blood glucose $\geq 140 \mathrm{mg} / \mathrm{dl}$ ) were more likely. An $\mathrm{SpO}_{2}<96.5 \%$ was nearly associated with an increased odds (OR 1.9; 95\% CI -0.03 to 3.8, $\mathrm{p}=0.054$ ); this finding requires confirmation with a larger sample size.

\section{DISCUSSION}

This study showed that lower nocturnal saturation was associated with worse glucose regulation in children with CF. In contrast to our hypothesis, however, there were no significant associations between parameters of sleep quality (either during polysomnography or during sleep in the home as measured by actigraphy) or markers of inflammation and glucose intolerance. To our knowledge, this is the first study to evaluate sleep and glucose disturbances in patients with CF.

\section{CF-related diabetes}

This study confirms that hyperglycaemia is common in children with CF. We studied subjects with no known diagnosis of diabetes, all of whom were at an age where annual diabetes screening by OGTT is often performed. A third of the subjects had either impaired glucose tolerance or CFRD without fasting hyperglycaemia. An additional $20 \%$ of subjects had abnormal glucose tolerance based upon a plasma glucose $>200 \mathrm{mg} / \mathrm{dl}$ at $60 \mathrm{~min}$, but were classified as normoglycaemic based upon the $2 \mathrm{~h}$ glucose level. ${ }^{23}$ The importance of glucose abnormalities in CF cannot be overemphasised. CFRD has been associated with worse pulmonary and nutritional status ${ }^{4}$ and worse clinical outcome with an earlier mortality. ${ }^{2}$ Fortunately, with early detection and aggressive diabetes treatment, survival has improved. ${ }^{1}$ The current study highlights the importance of not only identifying and treating CFRD as per published guidelines, but also considering earlier treatment of glucose abnormalities in $\mathrm{CF}$, and determining the underlying aetiology.

The pathophysiology of CFRD is not completely understood. CFRD is distinct from type 1 and type 2 diabetes mellitus, but shares features of both. ${ }^{2}$ Studies have demonstrated primarily decreased insulin secretion in CFRD, ${ }^{2}$ which arises in part from pancreatic islet destruction, although islet cell amyloid, a characteristic of type 2 diabetes, is present on pathological specimens. ${ }^{26}$ Insulin sensitivity in CF varies, with multiple factors relating to clinical status, and is decreased in the setting of impaired glucose tolerance ${ }^{3}$ and CFRD. ${ }^{2}$

\section{Saturation and glucose intolerance}

In this study, the subjects with CF were in overall good health, with a mean BMI $z$-score of 0.1 and mean $\mathrm{FEV}_{1}$ of $92 \%$ predicted. However, strictly speaking, $\mathrm{FEV}_{1}$ is a measure of forced air flow and not necessarily a measure of the current or ongoing infectious and/or inflammatory burden. Therefore, even though our subjects had a normal or mildly impaired $\mathrm{FEV}_{1}$, these data do not allow direct inference regarding the level of infection, inflammation or ongoing lung tissue damage that may have contributed to our data.

The mean nocturnal oxyhaemoglobin saturation was only slightly lower in the subjects with CF than in controls, and was still in the clinically normal range. In the absence of evidence of hypercapnia, the lower saturation was probably secondary to greater ventilation/perfusion mismatch in the subjects with CF as a result of decreased clearance of mucus plugs from the CF airway. Nevertheless, there was a clear association between lower baseline nocturnal saturation and glucose excursion. This is consistent with a recent study in a non-CF population showing that mild desaturations of $2-4 \%$ were associated with fasting hyperglycaemia. ${ }^{27}$

Clearly, the directionality of the relationship between lower saturation during sleep and hyperglycaemia cannot be deduced from this study. In fact, the finding of increased glucose excursion may only be an early marker of disease severity, a phenomenon that may not be accurately captured by pulmonary function tests. Decreased saturation resulting from increased small airways mucus plugging (that may not be detected by measurement of $\mathrm{FEV}_{1}$ ) could promote hyperglycaemia, as with other causes of nocturnal desaturation. ${ }^{27}$ Conversely, primary hyperglycaemia could impair airway neutrophil effectiveness, which could in turn promote small airways mucus plugging and hypoxaemia. Nonetheless, these findings offer a potential therapeutic window at a perhaps earlier time than that at which the more rapid decline in $\mathrm{FEV}_{1}$ associated with impaired glucose tolerance in CF is observed Improving nocturnal saturation may improve glucose excursion or, alternatively, nocturnal oxygen saturation may be improved by improving glucose excursion.

The current understanding of the relationship between nocturnal oxygen saturation and glucose intolerance is based mainly on research on obstructive sleep apnoea and insulin resistance. The nature of this relationship is different in the context of CF, which is not associated with sleep apnoea or obesity. However, artificial induction of hypoxaemia in nonobese, normal controls has also been shown to impair glucose tolerance. $^{828}$ The direct effect of hypoxaemia on glucose tolerance is not fully understood. Previous studies demonstrated that subjects exposed to hypoxia in the setting of high altitude had worsening glucose tolerance without concomitant rises in circulating levels of epinephrine. ${ }^{28}$ Sympathetic hyperactivity caused by hypoxaemia can affect glucose homeostasis by increasing glycogen breakdown and gluconeogenesis. Hypoxia can also induce a number of reactive mechanisms regulated by hypoxia-inducible factor 1, which induces expression of genes that encode glycolytic enzymes and glucose transporters. ${ }^{29}$

Evidence examining the effect of hypoxaemia on pancreatic $\beta$-cell function is sparse. A study of intermittent hypoxaemia and glucose tolerance in healthy adults showed that intermittent hypoxaemia was associated with decreased insulin sensitivity but had no effect on insulin secretion. ${ }^{8}$ However, animal studies suggest that hypoxaemia stimulates pancreatic $\beta$-cell replication, yet in the presence of hyperglycaemia, hypoxaemia leads to $\beta$-cell apoptosis. ${ }^{30}$ There is also evidence to suggest that apoptosis of human pancreatic islets correlates with hypoxiainducible factor 1 expression when exposed to hypoxia. ${ }^{31}$ It is therefore possible that hypoxaemia may affect both glucose utilisation and $\beta$-cell function in patients with CF. 
The gold standard for assessment of insulin sensitivity is the hyperinsulinaemic euglycaemic clamp, but this is timeconsuming and labour-intensive, and is not routinely performed in research studies on children due to ethical concerns. ${ }^{32-34}$ Furthermore, insulin sensitivity tends to be preserved in CF unless an individual is ill or has CFRD.

\section{Limitations}

This study was limited by our modest sample size. However, the relationship between nocturnal saturation and glucose was robust; this was verified by vigorous regression analysis accounting for multiple potential confounding factors. It is possible that measurement of oximetry over several nights would have strengthened the outcomes. We did not have actigraphy, OGTT and inflammatory markers for control subjects, which limits our interpretation of these findings with respect to the comparison between subjects with CF and normal, healthy subjects.

\section{Conclusion}

This study has shown that even mild decreases in nocturnal saturation were associated with impaired glucose regulation in children with CF. Further research is needed to determine the clinical importance of these findings.

Acknowledgements The authors would like to thank the patients and their families for participating in this study, as well as the sleep laboratory technologists, the Clinical and Translational Research Center nurses and David Stokes from the Translational Core Laboratory, and the physicians, nurses and staff from the Children's Hospital of Philadelphia Cystic Fibrosis Center. We thank Dr Elizabeth Fiorino for assistance in recruiting subjects.

Funding This study was supported by the National Institutes of Health: Junior Investigator Pilot Grant Program from UL1 RR024134; R01 HL58585.

\section{Competing interests None.}

Ethics approval This study was conducted with the approval of the Institutional Review Board of the Children's Hospital of Philadelphia.

Contributors All authors contributed to aspects of study design, data collection, data interpretation and manuscript review.

Provenance and peer review Not commissioned; externally peer reviewed.

\section{REFERENCES}

1. Moran A, Dunitz J, Nathan B, et al. Cystic fibrosis-related diabetes: current trends in prevalence, incidence, and mortality. Diabetes Care 2009;32:1626-31.

2. O'Riordan SM, Robinson PD, Donaghue KC, et al. Management of cystic fibrosis-related diabetes in children and adolescents. Pediatr Diabetes 2009:12(10 Suppl):43-50.

3. Hardin DS, Leblanc A, Marshall G, et al. Mechanisms of insulin resistance in cystic fibrosis. Am J Physiol Endocrinol Metab 2001;281:E1022-8.

4. Koch C, Rainisio M, Madessani U, et al. Presence of cystic fibrosis-related diabetes mellitus is tightly linked to poor lung function in patients with cystic fibrosis: data from the European Epidemiologic Registry of Cystic Fibrosis. Pediatr Pulmonol 2001:32:343-50.

5. Ip MS, Lam B, Ng MM, et al. Obstructive sleep apnea is independently associated with insulin resistance. Am J Respir Crit Care Med 2002;165:670-6.

6. Somers VK, Dyken ME, Clary MP, et al. Sympathetic neural mechanisms in obstructive sleep apnea. J Clin Invest 1995;96:1897-904.

7. Vgontzas AN, Chrousos GP. Sleep, the hypothalamic-pituitary-adrenal axis, and cytokines: multiple interactions and disturbances in sleep disorders. Endocrinol Metab Clin North Am 2002;31:15-36.
8. Louis M, Punjabi NM. Effects of acute intermittent hypoxia on glucose metabolism in awake healthy volunteers. J Appl Physiol 2009;106:1538-44.

9. Amin R, Bean J, Burklow K, et al. The relationship between sleep disturbance and pulmonary function in stable pediatric cystic fibrosis patients. Chest 2005; 128:1357-63.

10. Jankelowitz L, Reid KJ, Wolfe L, et al. Cystic fibrosis patients have poor sleep quality despite normal sleep latency and efficiency. Chest 2005;127:1593-9.

11. Tauman R, O'Brien LM, Gozal D. Hypoxemia and obesity modulate plasma C-reactive protein and interleukin-6 levels in sleep-disordered breathing. Sleep Breath 2007; 11:77-84.

12. Htoo AK, Greenberg $H$, Tongia $S$, et al. Activation of nuclear factor kappaB in obstructive sleep apnea: a pathway leading to systemic inflammation. Sleep Breath 2006;10:43-50.

13. El Sheikh M, Buckhalt JA, Granger DA, et al. The association between children's sleep disruption and salivary interleukin-6. J Sleep Res 2007:16:188-97.

14. Ohga E, Nagase T, Tomita T, et al. Increased levels of circulating ICAM-1, VCAM-1, and L-selectin in obstructive sleep apnea syndrome. J Appl Physiol 1999;87:10-14.

15. O'Brien LM, Serpero LD, Tauman R, et al. Plasma adhesion molecules in children with sleep-disordered breathing. Chest 2006;129:947-53.

16. Meier-Ewert HK, Ridker PM, Rifai N, et al. Effect of sleep loss on C-reactive protein, an inflammatory marker of cardiovascular risk. J Am Coll Cardiol 2004; 43:678-83.

17. Vgontzas AN, Zoumakis E, Bixler EO, et al. Adverse effects of modest sleep restriction on sleepiness, performance, and inflammatory cytokines. J Clin Endocrinol Metab 2004;89:2119-26.

18. Deepa R, Velmurugan K, Arvind K, et al. Serum levels of interleukin 6, C-reactive protein, vascular cell adhesion molecule 1 , and monocyte chemotactic protein 1 in relation to insulin resistance and glucose intolerance-the Chennai Urban Rural Epidemiology Study (CURES). Metabolism 2006;55:1232-8.

19. Nixon LS, Yung B, Bell SC, et al. Circulating immunoreactive interleukin-6 in cystic fibrosis. Am J Respir Crit Care Med 1998;157:1764-9.

20. Milla CE, Warwick WJ, Moran A. Trends in pulmonary function in patients with cystic fibrosis correlate with the degree of glucose intolerance at baseline. Am J Respir Crit Care Med 2000;162:891-5.

21. Melendres MC, Lutz JM, Rubin ED, et al. Daytime sleepiness and hyperactivity in children with suspected sleep-disordered breathing. Pediatrics 2004; 114:768-75.

22. Iber C, Ancoli-lsrael S, Chesson AL, et al. The AASM Manual for the Scoring of Sleep and Associated Events: Rule, Terminology and Technical Specifications. Westchster, IL: American Academy of Sleep Medicine, 2007.

23. Ode KL, Frohnert $B$, Laguna $T$, et al. Oral glucose tolerance testing in children with cystic fibrosis. Pediatr Diabetes 2010;11:487-92.

24. Moran A, Doherty L, Wang X, et al. Abnormal glucose metabolism in cystic fibrosis J Pediatr 1998:133:10-17.

25. Dobson L, Sheldon CD, Hattersley AT. Conventional measures underestimate glycaemia in cystic fibrosis patients. Diabet Med 2004;21:691-6.

26. Iannucci A, Mukai K, Johnson D, et al. Endocrine pancreas in cystic fibrosis: an immunohistochemical study. Hum Pathol 1984;15:278-84.

27. Stamatakis $\mathbf{K}$, Sanders $\mathbf{M H}$, Caffo B, et al. Fasting glycemia in sleep disordered breathing: lowering the threshold on oxyhemoglobin desaturation. Sleep 2008;31:1018-24.

28. Braun B, Rock PB, Zamudio $S$, et al. Women at altitude: short-term exposure to hypoxia and/or alpha(1)-adrenergic blockade reduces insulin sensitivity. J Appl Physiol 2001;91:623-31.

29. Iyer NV, Kotch LE, Agani F, et al. Cellular and developmental control of 02 homeostasis by hypoxia-inducible factor 1 alpha. Genes Dev 1998;12:149-62.

30. Yokoe T, Alonso LC, Romano LC, et al. Intermittent hypoxia reverses the diurnal glucose rhythm and causes pancreatic beta-cell replication in mice. J Physiol 2008;586:899-911.

31. Moritz W, Meier F, Stroka DM, et al. Apoptosis in hypoxic human pancreatic islets correlates with HIF-1alpha expression. FASEB J 2002;16:745-47.

32. Kopelman LM, Murphy TF. Ethical concerns about federal approval of risky pediatric studies. Pediatrics 2004;113:1783-9.

33. Code of Federal Regulations. 45 CFR 46:401-409. Additional protections for children involved as subjects in research. (Subpart D: US Department of Health and Human Services. In. Fed Reg). 1983:9816-20. http://ohsr.od.nih.gov/guidelines/ 45cfr46.html\#46.401.

34. Department of Health EaWNCftPoHSoBaBR. Research Involving Children Report and Recommendation. Washington, DC: Department of Health, Education and Welfare, (Publication no. [OS] 77-0004). 1977 